\title{
Delayed Diagnosis
}

\section{Atraumatic gluteal compartment syndrome}

\author{
J.E. Hynes and A. Jackson \\ Department of Diagnostic Radiology, Manchester Royal Infirmary, Oxford Road, \\ Manchester M13 9WL, UK
}

Summary: A case of atraumatic gluteal compartment syndrome complicated by sciatic nerve palsy and acute rhabdomyolysis is presented. A presumed diagnosis of deep venous thrombosis led to a delay in diagnosis. Gluteal compartment syndrome should be considered in the differential diagnosis of the swollen leg.

\section{Introduction}

Gluteal compartment syndrome (GCS) is rare and usually follows trauma or substance abuse with a prolonged period of immobility and/or reduced consciousness. Painful swelling of the involved muscles that increases on passive stretch is characteristic. Neurological symptoms due to nerve compression/ischaemia and crush syndrome are commonly associated. Measurement of tissue pressures within the affected compartment confirms the diagnosis and treatment is usually by fasciotomy.

A case is described in which GCS complicated a prolonged period of immobility. Non-surgical management led to a satisfactory outcome.

\section{Case report}

A 27 year old female was referred with a 36 hour history of painful, swollen right lower limb and a presumed diagnosis of deep venous thrombosis. Symptoms followed a prolonged sleep on the sitting room floor, prior to which she had taken $10 \mathrm{mg}$ of temazepam and two tablets of Diconal (dipipanone $10 \mathrm{mg}$, cyclizine $30 \mathrm{mg}$ ), which were prescribed for chronic pelvic inflammatory disease. On awakening, she experienced weakness in her right leg and foot which persisted, and was associated with increasing pain and swelling in the right thigh and buttock.

On examination, her vital signs were normal, there was tenderness and obvious swelling of the right leg. Her right mid-thigh measured $62 \mathrm{~cm}$, the left $56 \mathrm{~cm}$. The right mid-calf measured $38 \mathrm{~cm}$, the left $36 \mathrm{~cm}$. No obvious bruising or inflammation

Correspondence: J.E. Hynes, M.B., B.Ch., B.A.O., M.R.C.P.I., F.R.C.R.

Accepted: 15 September 1993 was seen and all pulses were easily palpable. Pain was elicited in the right buttock on hip flexion.

Neurological examination confirmed a sciatic nerve lesion with sensory deficit, absent ankle reflex and a foot drop on the right side.

Ultrasound of her kidneys was normal except for increased echogenicity in the renal pyramids. Ultrasound of the pelvic and lower limb veins was normal. Biochemistry investigations demonstrated acute renal failure (urea, $21 \mathrm{mmol} / \mathrm{l}$; creatinine, $320 \mu \mathrm{mol} / 1$; potassium, $4.6 \mathrm{mmol} / \mathrm{l})$. Haematological investigations demonstrated a leucocytosis of $18.0 \times 10^{9} / 1$, haemoglobin, $13.0 \mathrm{~g} / \mathrm{dl}$.

A presumed diagnosis of deep venous thrombosis (DVT) was made and she was treated by systemic anticoagulation using heparin $(24,000 \mathrm{U} /$ 24 hours). Creatine kinase levels (46,000 IU/1) were grossly elevated leading to the diagnosis of acute renal failure secondary to rhabdomyolysis (crush syndrome).

Considering the history and clinical signs, this was felt to be due to an atraumatic gluteal compartment syndrome secondary to prolonged immobility. The heparin was discontinued immediately and her renal failure was treated conservatively. The patient became anuric and creatinine levels rose to $1,100 \mu \mathrm{mol} / 1$. Spontaneous diuresis commenced 5 days following admission and her renal function returned to normal by one month of follow-up.

Four months following discharge clinical examination and nerve conduction studies demonstrated persistent sensory loss consistent with involvement of the sciatic nerve on the right side. Conduction studies showed some loss of axonal function with delay of conduction in the sciatic nerve proximally on the right but no evidence of a common peroneal lesion. Motor responses also showed signs of axonal degeneration with early signs of re-inner- 
vation in the anterior and posterior compartments. A good recovery was predicted on the basis of these tests.

\section{Discussion}

A compartment syndrome results when increased pressure within an enclosed space, such as a fascial or osteo-fascial compartment, causes the circulation and function of the tissues in that space to be compromised. ${ }^{1}$ Common causes include trauma and a prolonged period of recumbency or unconsciousness as may occur with alcohol and substance abuse or general anaesthesia. ${ }^{2}$ Other recognized associations include severe exertion and anticoagulation treatment. $^{3}$

Compartment syndromes have been described in various fascial and osteofascial compartments in the upper and lower limbs, and even skin has been described as a limiting boundary. ${ }^{4}$ The diagnosis can be made on the basis of the clinical signs and symptoms which include weakness of the affected muscles, pain out of proportion to that expected for the clinical situation, with increased pain on passive stretch, tenseness of the affected compartment, and sensory and motor disturbance in the distribution of nerves passing through the involved compartment. ${ }^{5}$ Symptoms progress through paraesthesiae paresis, anaesthesia and finally paralysis with loss of peripheral pulses. ${ }^{6}$ The diagnosis can be confirmed by measurement of the elevated compartment tissue pressure by the use of a wick catheter ${ }^{5,7}$ Pressures between 30 and $50 \mathrm{mmHg}$ may produce muscle ischaemia and necrosis will occur if pressure is maintained at these levels for 4-8 hours. ${ }^{2}$

The time interval between the precipitating event and the onset of symptoms is usually $10-15$ hours but it may be as long as 6 days. ${ }^{2}$ Both the duration and magnitude of the pressure rise are important factors in the production of soft tissue injury. ${ }^{8}$ Hargens et al. showed that the time required to produce peripheral nerve conduction block is inversely proportional to intracompartmental pressure. ${ }^{5}$ In this study nerve conduction was blocked completely in less than 2 hours at pressures between

\section{References}

1. Matsen, F.A., III. Compartmental syndrome: a unified concept. Clin Orthop 1975, 113: 8.

2. Rommel, F.M., Kabler, R.L. \& Mowad, J.J. The crush syndrome: a complication of urological surgery. J Urol 1986, 135: 809-811.

3. Hay, S.M. \& Allen M.J. Acute compartment syndromes resulting from anticoagulant treatment. $\mathrm{Br}$ Med $J$ 1992, 305: 1474-1475.

4. Gaspard, D.G. \& Kohl, R.D. Compartmental syndromes in which skin is the limiting boundary. Clin Orthop 1975, 113: 65-68.
80 and $120 \mathrm{mmHg}$, and fasciotomy was recommended when pressures exceed $30 \mathrm{mmHg}$ or the duration of symptoms exceeds 6-8 hours. The crush syndrome describes the systemic manifestations of rhabdomyolysis following a compartment injury, including myoglobinuria, hyperkalaemia and acidosis which may lead to renal failure, shock, cardiac arrhythmia and death. ${ }^{2,9}$

Gluteal compartment syndrome is rare and is usually associated with the precipitating causes described above. Other rarer causes include sickle cell trait, ${ }^{2}$ intramuscular injection, ${ }^{10}$ spontaneous gluteal artery rupture, ${ }^{11}$ and prolonged surgical procedures associated with the lateral decubitus, lithotomy and sitting positions. ${ }^{2}$ A case of bilateral gluteal compartment syndrome has been described in a patient following a craniotomy in the sitting position. ${ }^{12}$

Owen et al. described the anatomy of the gluteal compartment and its role in the compartment syndrome. ${ }^{13}$ The gluteal compartment is enclosed by the fascia lata of the thigh. This splits into two layers to surround the muscle bellies of the gluteus maximus posteriorly and the tensor fascia lata anteriorly. Between these muscles the combined fascia overlies the gluteus medius and minimus. This region functions as three separate compartments and surgical treatment must consider each of these functionally separate compartments. The sciatic nerve does not lie within the fascial envelope except in cases where it perforates the piriformis but the nerve is vulnerable to compression by swelling in the adjacent muscles.

Owing to the large muscle mass gluteal compartment syndrome is usually accompanied by the crush syndrome. However, owing to a low clinical awareness, the variability of the presenting complaints and the presence of severe metabolic abnormalities, the diagnosis is frequently delayed or missed. Such a delay was seen in the case presented and the misdiagnosis of a DVT with commencement of anticoagulation therapy could have had a detrimental effect.

As first suggested by Rommel et al., ${ }^{2}$ and Small et $a l .{ }^{14}$ the differential diagnosis of painful swelling in the lower extremities presenting as a DVT should include compartment syndrome.
5. Hargens, A.R., Romine, J.S., Sipe, J.C., Evans, K.L., Mubarak, S.J. \& Akeson, W.H. Peripheral nerve conduction block by high muscle compartment syndrome. J Bone Joint Surg 1979, 61A: 192-200.

6. Schmalzreid, T.P., Neal, W.C. \& Eckardt, J.J. Gluteal compartment and crush syndromes. A report of three cases and review of the literature. Clin Orthop 1992, 277: 161-165.

7. Whitesides, T.E., Haney, T.C., Morimoto, K. \& Harada, H. Tissue pressure measurements as a determinant for the need of fasciotomy. Clin Orthop 1975, 113: 43-51. 
8. Sheridan, G.W., Matsen, F.A., III \& Krugmire, R.B. Further investigations on the pathophysiology of the compartment syndrome. Clin Orthop 1977, 123: 266-270.

9. Mubarak, S.J. \& Owen, C.A. Compartmental syndrome and it's relation to the crush syndrome: A spectrum of disease. Clin Orthop 1975, 113: 81-89.

10. Lund, N. \& Jensen, K.H. Glutealt kompartmentsyndrom. Ugeskr Laeger 1990, 152: 610-611.

11. Schmalzried, T.P. \& Eckardt, J.J. Spontaneous gluteal artery rupture resulting in compartment syndrome and sciatic neuropathy. Clin Orthop 1992, 275: 253-257.
12. Poppi, M., Giuliani, G., Gambari, P.I., Acciarri, N., Gaist, G. \& Calbucci, F. A hazard of craniotomy in the sitting position: the posterior compartment of the thigh. Case report. J Neurosurg 1989, 71: 618-619.

13. Owen, C.A., Woody, P.R., Mubarak, S.J. \& Hargens, A.R. Gluteal compartment syndromes. A report of three cases and management utilizing the Wick catheter. Clin Orthop 1978, 132: $57-60$.

14. Small, M., Alzaid, A. \& Gray, H.W. Rhabdomyolysis mimicking deep vein thrombosis. Postgrad Med J 1987, 63: $653-655$. 